\title{
Analyse de l'instabilité par flambage des couches à la mine de Grand-Baume, Charbonnages de France
}

\section{Analysis of a strata buckling mechanism at the Grand-Baume coal mine, Charbonnages de France}

\author{
P. CHOQUET, J. HADJIGEORGIOU \\ Université Laval, Département des mines et métallurgie \\ P. MANINI, E. MATHIEU, V. SOUKATCHOFF \\ Ecole des Mines de Nancy - Laboratoire de mécanique des terrains, INERIS**

\section{Y. PAQUETTE} \\ INERIS, Groupe géotechnique et atmosphères industrielle *
}

Rev. Franç. Géotech. $\mathrm{n}^{\circ} 60$, pp. $61-70$ (juillet 1992)

\section{Résumé}

La communication décrit un mécanisme de flambage des couches rocheuses qui pourrait se produire le long du flanc ouest de la mine à ciel ouvert de GrandBaume au moment où la profondeur maximale d'exploitation sera atteinte.

Afin d'étudier ce mécanisme de rupture, deux méthodes d'analyse ont été utilisées, d'une part des essais en modèle réduit sur table à frottement et d'autre part la modélisation mathématique par un programme de calcul à l'équilibre limite du flambage à trois pivots. Le détail de la mise en œuvre de ces deux méthodes de même que leur confrontation au cas de la mine de Grand-Baume sont décrits dans I'article.

\section{Abstract}

The paper describes a three hinge strata buckling mechanism and its influence on the west wallof the Grand-Baume open pit coal mine. The analysis is extended to investigate the stability at the projected maximum depth of the pit.

In order to study and quantify the failure mechanism, two analysis methods were employed. The first method used the base friction table on which scale models of the slope were represented. The second method made use of the mathematical modelling of the slope stability by means of a limit equilibrium computer program, specific to three hinge strata buckling.

Details on the application and the results of the two methods are presented. These are henceforth compared with the field observations at the Grand-Baume mine.

- QUE G1X 794 Québec, Canada.

* Parc de Saurupt, 54042 Nancy Cedex. 


\section{INTRODUCTION}

La mine de Grand-Baume située dans le Gard (France), au nord de la Grand'Combe, est exploitée depuis environ huit années. Il s'agit d'une fosse ayant environ $1 \mathrm{~km}$ de longueur et $0,4 \mathrm{~km}$ de largeur subdivisée en quatre fosses numérotées 1 à 4 . Les fosses 1 à 3 ont été exploitées successivement suivant un axe sud-ouest/nord-est jusqu'à une profondeur approximativement de $150 \mathrm{~m}$ et sont maintenant remblayées. Le côté ouest des fosses est formé d'un pli couché dont le flanc supérieur a un pendage de l'ordre de $40^{\circ}$ (fig. 1), de telle sorte que le talus ouest adopte l'inclinaison des couches formées de grès et de siltites d'âge Stéphanien moyen.

La fosse 4, en cours d'exploitation, atteint actuellement (1er janvier 1991) une profondeur de $150 \mathrm{~m}$, soit approximativement la profondeur atteinte par les fosses 1 à 3 . Il est prévu d'exploiter la fosse 4 jusqu'à une profondeur de $250 \mathrm{~m}$ pour récupérer la charnière du pli dans laquelle la couche de charbon s'épaissit.

Dans les fosses 2 et 3 , des glissements superficiels de quelques mètres d'épaisseur des couches schisteuses constituant le talus ouest ont été observés en fin d'exploitation des fosses. Ces glissements ont été décrits et analysés par SOUKATCHOFF et al. (1991). L'occurrence de ces glissements de petite envergure a toutefois fait craindre que des glissements à plus grande échelle puissent se produire vers la fin de l'exploitation de la fosse 4 , au moment où la charnière du pli sera dégagée. Ce dégagement aura pour effet de libérer la base des couches de leur butée à un endroit particulièrement critique puisque la courbure concave des couches (fig. 2) au-dessus de la charnière est a priori défavorable à la stabilité. Face à cette situation, un soutènement par boulons câbles a été dimensionné et mis en place sur le talus ouest de la fosse. Les performances de ce soutènement ne sont toutefois pas discutées dans cette communication.

Le but de cette communication est de décrire deux approches: l'utilisation d'un modèle physique puis d'un logiciel, ayant servis à étudier en détail le mécanisme de rupture qui pourrait affecter le talus ouest de la fosse 4 , lorsque celle-ci aurai atteint ses limites ultimes. Le mécanisme de rupture est un flambage des couches de roche situées dans la partie inférieure de la fosse, sous l'effet de la poussée apportée par la partie supérieure de ces mêmes couches.

\section{MÉCANISME DU FLAMBAGE DES COUCHES}

Le flambage des couches est un mécanisme qui ressemble à celui que l'on interprète normalement par la théorie de flambage des poutres d'Euler. Ainsi, une partie active d'un banc de roche exerce une force qui provoque le flambage d'une partie passive sous-

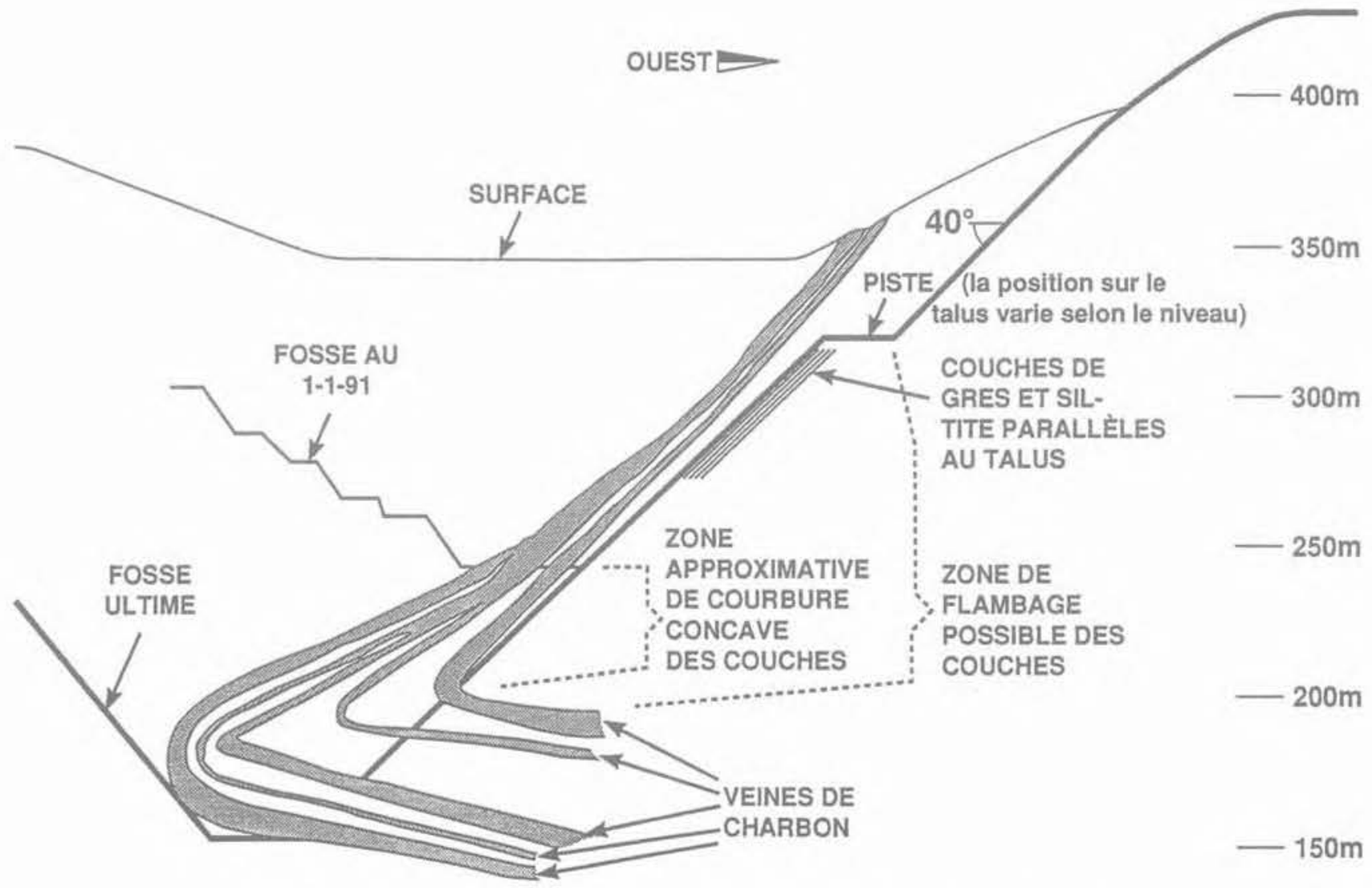

Fig. 1. - Coupe transversale du talus ouest de la fosse 4 au niveau 95.

Fig. 1. - Transversal section of west wall of pit 4 at level 95. 


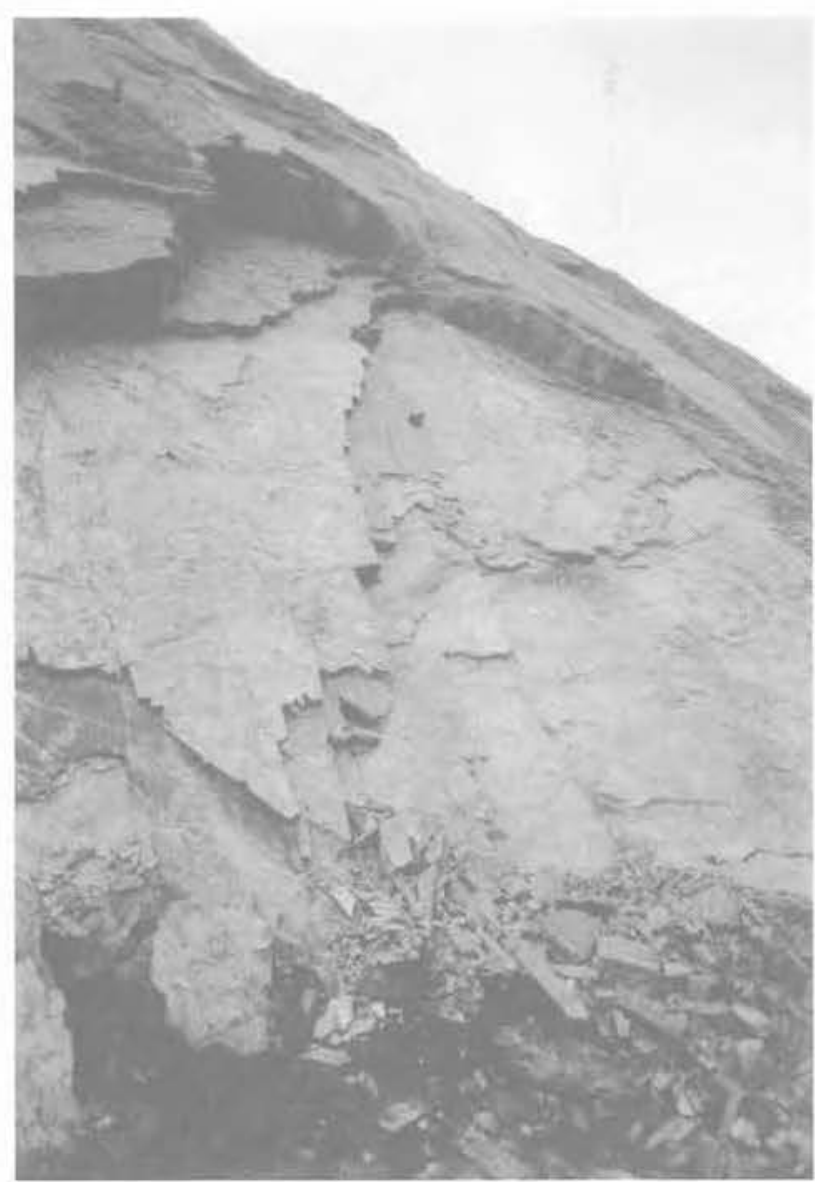

Fig. 2. - Couches de grès à l'élévation $250 \mathrm{~m}$ du talus ouest (noter la courbure concave des couches vers l'intérieur de la fosse).

Fig. 2. - Sandstone layers at elevation $250 \mathrm{~m}$ of West Wall (note the concave curvature of lavers towards inside of pit).

jacente. Lorsque la force exercée par la partie active est suffisante, il y a rupture, c'est-à-dire expulsion vers l'extérieur des couches de roche, tel qu'illustré à la figure 3 .

En règle générale, la théorie d'Euler ne permet pas à elle seule d'expliquer l'occurrence du flambage et

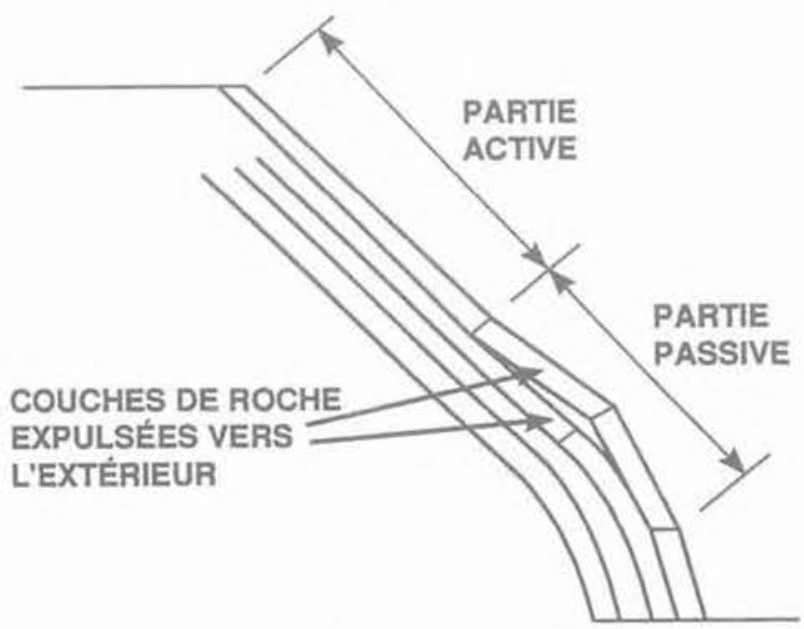

Fig. 3. - Mécanisme de rupture par flambage des couches. Fig. 3. - Failure mechanism by strat buckling. de la rupture. Les couches ont de façon naturelle un certain rayon de courbure alors que les formules d'Euler qui correspondent à la théorie classique du flambage des poutres et que l'on retrouve dans tous les livres de référence en résistance des matériaux, ont été développées en supposant les couches parfaitement rectilignes. Les formules d'Euler ne tiennent pas compte des moments fléchissants engendrés par les forces agissant à l'extérieur de l'axe des couches et qui amplifient les effets de flexion. Ainsi, l'application des seules formules d'Euler à un cas particulier où les couches présentent une certaine courbure pourrait mener à prédire la stabilité pour une situation qui en réalité mènera à la rupture.

Un modèle de calcul à l'équilibre limite s'appliquant aux couches non planes a été proposé par CAVERS (1981). Par la suite, dans le cadre d'un contrat de recherche de CANMET (Ministère de l'Energie. Mines et Ressources, Canada), CAVERS a préparé un code de calcul appelé Curvbuk (CAVERS, 1988 ; CANMET, 1986) permettant de réaliser l'analyse de stabilité des couches face au flambage. La version source du code de calcul est disponible (CANMET, 1986).

Le logiciel Curvbuk a été utilisé par les auteurs afin d'étudier le modèle géométrique de la figure 3 en ladaptant à la géométrie particulière du flanc ouest de la fosse de Grand-Baume. Afin de corroborer les résultats obtenus à laaide du logiciel, une étude en modèle réduit par table à frottement décrite dans le paragraphe suivant a aussi été réalisée.

\section{3. ÉTUDE EN MODĖLE RÉDUIT PAR LA TABLE À FROTTEMENT}

La table à frottement permet d'étudier le comportement d'un massif rocheux sur un modèle réduit (HOBBS, 1966 : SPANG, 1976; EGGER et al., 1979). Cet appareil est basé sur le principe du frottement de base, qui permet le placement des forces de gravité dans un modèle physique à deux dimensions par des forces de frottements à la base de celuici. Ce principe a été mis en équation par BRAY et GOODMAN (1981). Le modèle est soumis à un mouvement de translation par le biais d'une large courroie continue en papier sablé, sur laquelle il repose, et qui est entraîné par un petit moteur à faible vitesse constante (fig. 4). Ce mouvement de translation est par la suite restreint par une barrière fixe installée au bout approprié de la table, immobilisant ainsi le modèle tout en permettant le déplacement de la courroie sous celui-ci. Le frottement exercé par la courroie sur le modèle engendre sur la barrière fixe une force permettant de simuler ainsi la gravité. La table à frottement permet de bien visualiser ce qui pourrait se passer en réalité. Pour utiliser ce mécanisme, il faut au préalable trouver le bon matériau équivalent, destiné à remplacer le matériau réel que l'on désire représenter à plus petite échelle.

\subsection{Choix du matériau équivalent}

Le matériau doit vérifier des équations de similitude dont la plus importante est la suivante (MANDEL, 1962) :

$$
\frac{\sigma_{e}}{\sigma_{r}}=\frac{\rho_{e} g_{e} l_{e}}{\rho_{r} g_{r} l_{r}}
$$




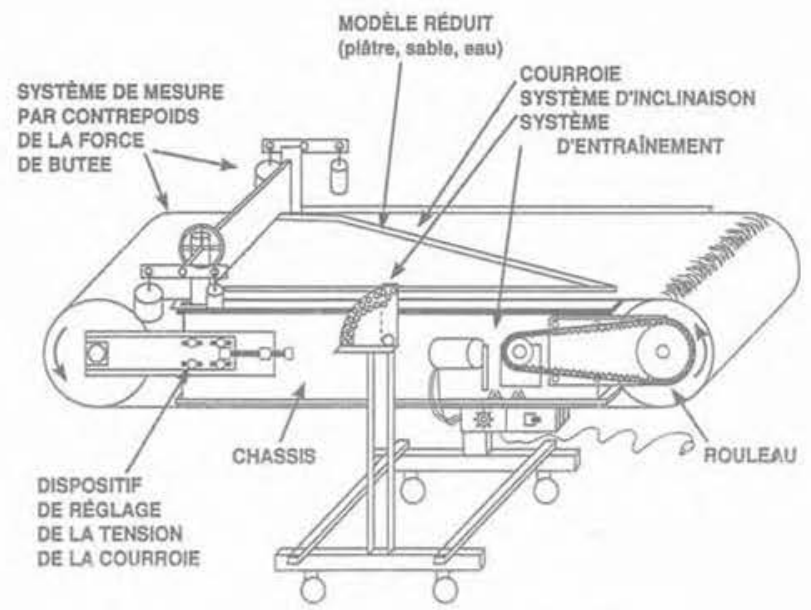

Fig. 4. - Schéma de la table à frottement. Fig. 4. - Schematic of friction table.

avec : $\sigma$ : tenseur des contraintes,

$\rho$ : masse volumique,

$\mathrm{g}$ : force de masse, par unité de volume,

1 : longueur,

e : désignant le matériau équivalent,

$r$ : désignant le matériau réel.

Le matériau sélectionné pour lapplication présente est un mélange de plâtre, sable et eau. Les proportions en poids sont les suivantes: $60 \%$ de sable, $25 \%$ d'eau et $15 \%$ de plâtre. Des échantillons de $4,1 \mathrm{~cm}$ de diamètre et $8,2 \mathrm{~cm}$ de longueur ont d'abord été moulés pour vérifier les propriétés mécaniques du matériau.

Dix-sept échantillons ont été préparés en utilisant des proportions identiques mais en faisant varier le temps d'étuvage de 5 heures à 48 heures. La masse volumique des échantillons a également été déterminée.
Les tableaux des figures 5 et 6 résument les caractéristiques des échantillons et les résultats des essais de compression uniaxiale et des essais de traction effectués. Sur ces tableaux, on a aussi ajouté la valeur de la résistance en compression et en traction du matériau réel que le matériau équivalent permet de représenter par application de l'équation 1 , en tenant compte des paramètres suivants :

$\rho_{e}=1,48$ (moyenne de tous les échantillons préparés) ;

$\rho_{\mathrm{r}}=2,50$ (masse volumique du grès) ;

$\frac{\mathrm{l}_{\mathrm{e}}}{\mathrm{l}_{\mathrm{f}}}=180$ (facteur d'échelle géométrique choisi en tenant compte des dimensions de la table à frottement et du talus à modéliser).

Le rapport $\frac{\sigma_{e}}{\sigma_{\mathrm{r}}}$ obtenu est alors de 106,6 et a été utilisé dans les figures 5 et 6 .

En observant les résultats des tableaux, il s'avère que plus les échantillons sont étuvés et plus leur résistance en compression diminue, sans toutefois que la variation ne soit excessivement importante. Une durée d'étuvage de 24 heures a en conséquence été choisie pour la construction des modèles réduits décrits au paragraphe suivant.

En ce qui concerne les essais de résistance en traction, les résultats obtenus ont été, semble-t-il, entachés d'erreur par le fait que deux essais seulement ont été réalisés pour chaque durée d'étuvage sur les deux moitiés d'un même échantillon de $8,2 \mathrm{~cm}$ de longueur. Ainsi, tel qu'indiqué à la figure 6 , la partie haute de l'échantillon placée dans l'étude a chaque fois donné une résistance en traction plus faible que la partie basse du même échantillon. On peut expliquer ce phénomène par l'accumulation de l'eau dans l'échantillon du bas qui contribue à en rehausser la résistance.

\begin{tabular}{|c|c|c|c|}
\hline $\begin{array}{c}\text { Durée d'étuvage } \\
\text { (heures) }\end{array}$ & $\begin{array}{c}\text { Masse volumique } \\
\left(\mathrm{g} / \mathrm{cm}^{3}\right)\end{array}$ & $\begin{array}{c}\text { Résistance en compression } \\
\text { (MPa) }\end{array}$ & $\begin{array}{c}\text { Réstance } \\
\text { compression } \\
\text { matériau réel (MPa) }\end{array}$ \\
\hline 5 & 1,49 & 0,42 \\
5 & 1,53 & 0,60 \\
& 1,55 & 0,51 & \\
\hline Moyenne & 1,53 & 0,51 & 54,4 \\
\hline 24 & 1,54 & 0,53 & \\
\hline 24 & 1,51 & 0,49 & \\
24 & 1,48 & 0,48 & 52,2 \\
\hline Moyenne & 1,53 & 0,49 & \\
\hline 48 & 1,52 & 0,39 & \\
\hline 48 & 1,45 & 0,46 & \\
\hline 48 & 1,48 & 0,40 & \\
\hline Moyenne & 1,44 & 0,55 & \\
\hline
\end{tabular}

Figure 5. - Résultats des essais de résistance en compression sur le matériau équivalent. Figure 5. - Results of compressive strength tests on the equivalent material. 


\begin{tabular}{|c|c|c|c|}
\hline $\begin{array}{c}\text { Durée d'étuvage } \\
\text { (heures) }\end{array}$ & $\begin{array}{c}\text { Masse volumique } \\
\left(\mathrm{g} / \mathrm{cm}^{3}\right)\end{array}$ & $\begin{array}{c}\text { Résistance en compression } \\
\text { (MPa) }\end{array}$ & $\begin{array}{c}\text { Résistance } \\
\text { en traction } \\
\text { matériau réel (MPa) }\end{array}$ \\
\hline 5 & 1,47 & 0,099 & \multirow{2}{*}{19,5} \\
\hline 5 & 1,55 & 0,266 & \multirow{2}{*}{} \\
\hline Moyenne & 1,51 & 0,183 & \multirow{2}{*}{9,7} \\
\hline 24 & 1,37 & 0,049 & \\
\hline Moyenne & 1,45 & 0,133 & \\
\hline 48 & 1,41 & 0,091 & 10,02 \\
\hline 48 & 1,43 & 0,066 & \\
\hline Moyenne & 1,45 & 0,121 & \\
\hline
\end{tabular}

Figure 6. - Résultats des essais de résistance en traction sur le matériau équivalent.

Figure 6. - Results of tensile strength tetst on the equivalent material.

L'objectif recherché lors de la sélection du matériau équivalent était de reproduire le matériau réel constitué de couches de grès et siltites. Bien que ne disposant pas de résultats d'essais de compression unixaxiale ou de traction sur ces roches, une valeur de $50 \mathrm{MPa}$ avait préalablement été jugée représentative, par les auteurs, de leur résistance en compression réelle sur le terrain. La sélection du matériau équivalent a ensuite été faite en recherchant un dosage d'ingrédients tel que la résistance en compression du matériau réel représenté soit approximativement égale à cette valeur.

\subsection{Réalisation des essais}

Il s'est avéré nécessaire de simplifier la géométrie du flanc ouest à modéliser à cause de la faible échelle de $1 / 180$ du modèle, mais aussi dans le but de reproduire des géométries similaires à celles qui devraient par la suite être analysées par le logiciel Curvbuk. La figure 7 illustre la géométrie du modèle

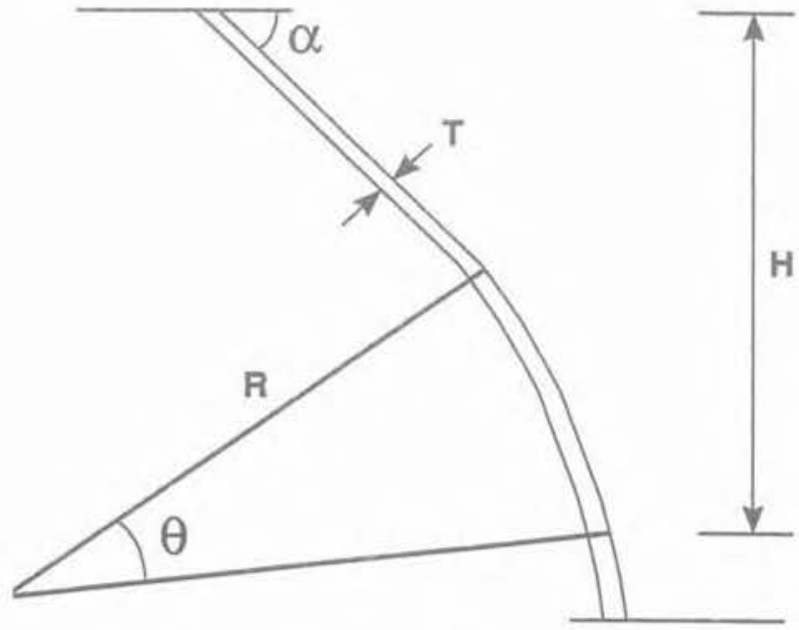

Fig. 7. - Modèle géométrique pour étude en modèle réduit par la table à frottement.

Fig. 7. -Geometrical model for reduced scale study with the friction table. qui a été retenue de même que la définition des paramètres qui sont le pendage $\alpha$, le rayon de courbure $\mathrm{R}$, l'angle de courbure $\theta$, l'épaisseur du banc $\mathrm{T}$ et la hauteur $\mathrm{H}$.

Le rayon de courbure $\mathrm{R}$ a été le même pour tous les modèles, soit $28 \mathrm{~cm}$, ce qui représente $50 \mathrm{~m}$ dans la réalité. Cette valeur est représentative du rayon de courbure réel des couches près du pied du flanc ouest. D'autre part, l'épaisseur des modèles a été arbitrairement choisie à $2 \mathrm{~cm}$. Toutefois, ce dernier paramètre n'influence pas la valeur de la gravité simulée dans le modèle tel qu'on peut le déduire des équations théoriques du principe de la table à frottement (BRAY et GOODMAN, 1981). En ce qui concerne la hauteur $H$, elle a varié selon l'espace disponible sur la table à frottement entre $75 \mathrm{~cm}$ et $88 \mathrm{~cm}$, ce qui représente 135 à $158 \mathrm{~m}$ de hauteur.

Le moulage des modèles a été réalisé de la façon suivante. Il faut déterminer les quantités des différents produits selon les proportions et la dimension du modèle. On verse l'eau dans un mélangeur, puis le sable. Le platre est ensuite versé progressivement dans le mélangeur pour éviter la formation de grumeaux. Le mélange est coulé dans le moule, mis dans un four et chauffé à $125^{\circ} \mathrm{C}$ pendant $24 \mathrm{~h}$. Ensuite, le modèle est démoulé et placé sur la table à frottement.

Onze moulages représentant autant de configurations différentes décrites au tableau de la figure 8 ont été effectués. Il est à noter que chaque configuration a pu être soumise à l'essai trois fois en découpant dans le modèle un nouveau banc parallèle au premier après chaque essai. Les résultats des essais sont également présentés sur la figure 8.

La hauteur indiquée sur la figure 8 est la hauteur $H$ du modèle au moment des essais. Cette hauteur est très importante car plus la hauteur est grande, plus il y a de l'instabilité. Or, avec la table à frottement, la hauteur est limitée par la grandeur du moule et de la table. Ainsi lorsque le modèle est stable, on peut conclure que tous les modèles qui ont une hauteur plus petite seront stables, par contre dans le cas d'une instabilité, on peut dire que tous les modèles qui ont 


\begin{tabular}{|c|c|c|c|c|c|c|c|}
\hline & & & \multicolumn{2}{|c|}{ Modèle } & \multicolumn{2}{|c|}{ Talus réel } & \multirow{2}{*}{ Commentaires } \\
\cline { 4 - 6 } Configuration & $\alpha$ (degrés) & $\theta$ (degrés) & $T(\mathrm{~cm})$ & $\mathrm{H} \mathrm{(cm)}$ & $\mathrm{T}(\mathrm{m})$ & $\mathrm{H}(\mathrm{m})$ & \\
\hline 1 & 40 & 25 & 1 & 77 & 1,8 & 138 & Stable \\
2 & 40 & 25 & 1,5 & 75 & 2,7 & 135 & Stable \\
3 & 45 & 30 & 1 & 88 & 1,8 & 158 & Rupture \\
4 & 45 & 30 & 1,5 & 87 & 2,7 & 157 & Stable \\
5 & 45 & 30 & 2 & 84 & 3,6 & 151 & Stable \\
6 & 50 & 25 & 1 & 88 & 1,8 & 158 & Rupture \\
7 & 50 & 25 & 1,5 & 88 & 2,7 & 158 & Stable \\
8 & 50 & 25 & 2 & 84 & 3,6 & 151 & Stable \\
9 & 55 & 30 & 1 & 88 & 1,8 & 158 & Rupture \\
10 & 55 & 30 & 1,5 & 80 & 2,7 & 144 & Rupture \\
11 & 55 & 30 & 2 & 82 & 3,6 & 148 & Stable \\
\hline
\end{tabular}

Figure 8 - Résuitats des essais sur la table à frottement. - Figure 8 , - Results of tests with the friction table.

une hauteur plus grande seront instables. Les figures 9 et 10 présentent différents essais obtenus avec la table à frottement. La figure 9 est un essai fait sur la configuration 6 ayant un pendage de $50^{\circ}$, un angle de courbure de $25^{\circ}$ et un rayon de courbure réel de $50 \mathrm{~m}$. L'épaisseur du banc est de $1 \mathrm{~cm}$, ce qui représente $1,8 \mathrm{~m}$, la base du modèle fait $80 \mathrm{~cm}$. A l'endroit de la courbure du banc, il y a eu gonflement puis rupture du banc. De plus, on observe qu'il est possible d'évaluer la longueur L1 du bloc supérieur qui est un paramètre important dans la modélisation par Curvbuk présentée au paragraphe suivant. Cette observation constitue en soi un apport de très grande utilité qui justifie l'utilisation de la table à frottement. La figure 10 représente un essai fait sur la configuration 3 ayant un pendage de $45^{\circ}$ et un angle de courbure de $30^{\circ}$. La rupture s'est faite également au niveau de la courbure et on peut remarquer la longueur L1 du bloc supérieur qui est plus petite que celle de la figure 9. La hauteur des deux modèles est de $88 \mathrm{~cm}$, ce qui représente $158 \mathrm{~m}$; le talus de la mine de Grand-Baume atteindra une hauteur supérieure lorsque le coude de la couche de charbon sera enlevé.

En conclusion, l'utilisation de la table à frottement laisse entrevoir qu'il y a possibilité de rupture par flambage des couches au moment où le fond de fosse atteindra l'élévation $200 \mathrm{~m}$ (fig. 1), puisqu'à ce moment la hauteur du talus dépassera $200 \mathrm{~m}$ du sommet à la base. Les configurations 1 et 2 avec $\alpha=40^{\circ}$ qui est la valeur la plus proche du véritable pendage du talus n'ont pas atteint la rupture lors des essais, toutefois, les autres configurations avec $\alpha$ $=45^{\circ}$ à $55^{\circ}$ ont présenté des cas de rupture qui apparaissent principalement influencés par l'épaisseur $\mathrm{T}$ des couches. Ainsi, la configuration 3 indique que pour une épaisseur des couches inférieure à $1,8 \mathrm{~m}$, la rupture serait atteinte dans le talus. Des essais supplémentaires de configurations avec $\alpha=40^{\circ}$ seraient nécessaires afin de cerner la valeur de l'épaisseur des couches au-dessous de laquelle la rupture serait possible afin de comparer cette valeur à l'épaisseur véritable des couches de grès et de siltite à la mine de Grand-Baume. D'autre part, il faut souligner que malgré sa grande utilité pour visualiser les mécanismes de rupture, la table à frottement ne permet pas de tenir compte de l'effet des pressions interstitielles d'eau dans le talus qui peuvent considérablement modifier la stabilité de ce dernier.

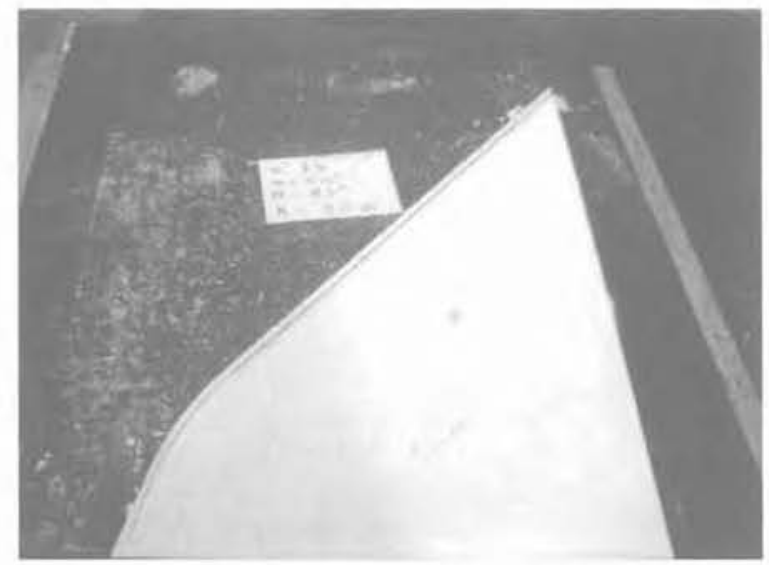

Avant l'essai. - Before test.

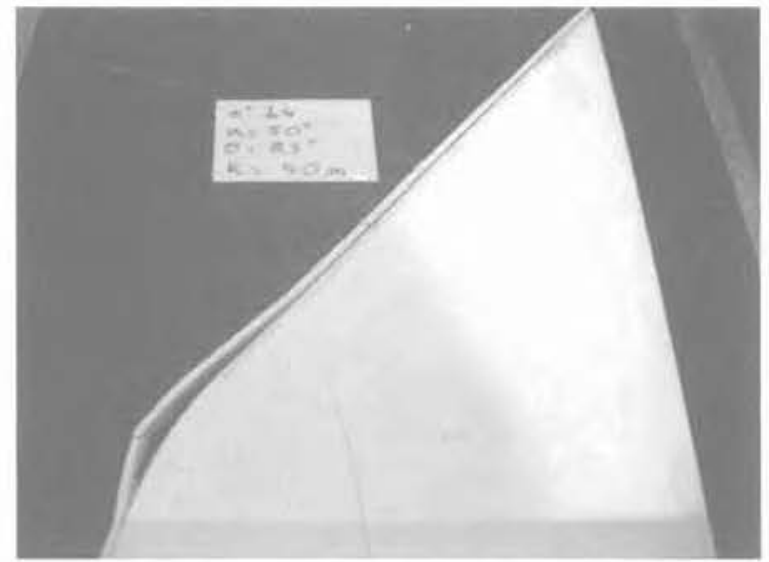

Rupture observée après Y'essai. - Failure observed after test.

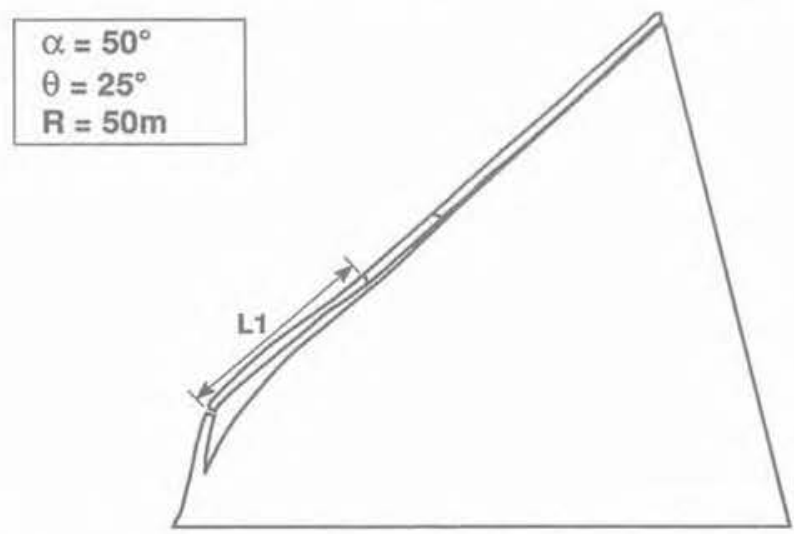

Schéma du modàle rupturé. - Schematic of model at failure.

Fig. 9. - Essai sur la configuration 6. Fig. 9. - Test on configuration 6 . 


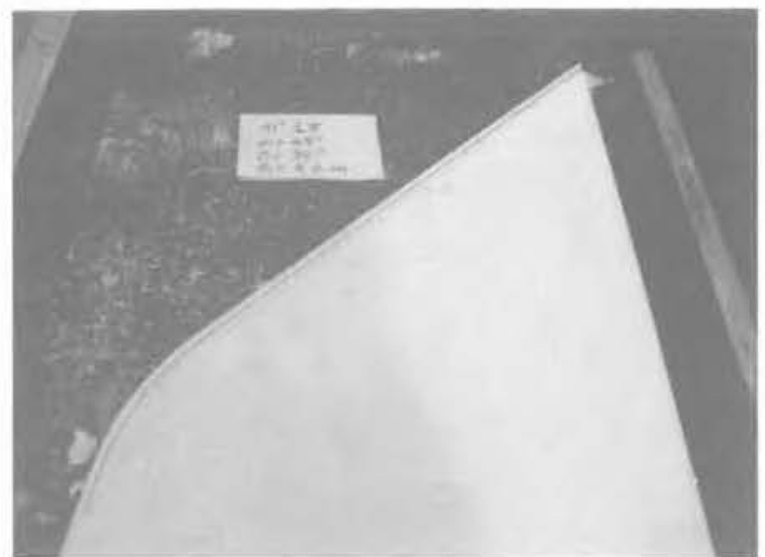

Avant l'essai. - Before test.

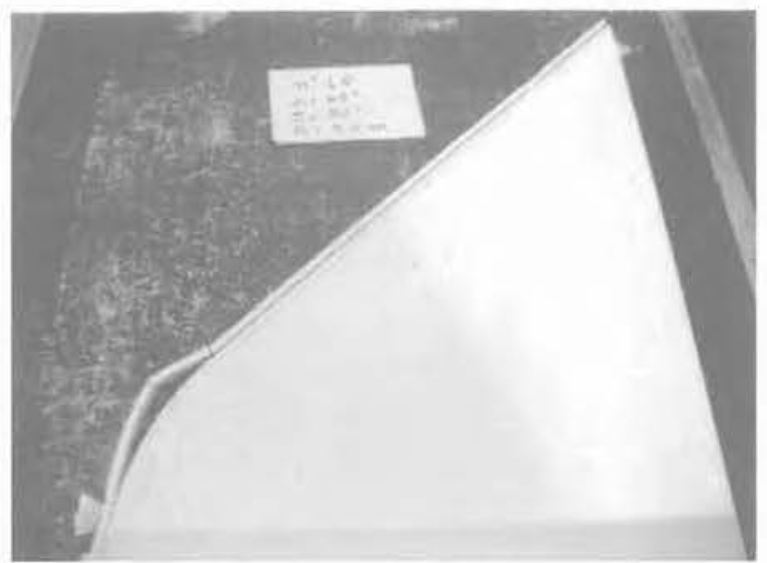

Rupture observée après I'essai. - Failure observer after test.

$\alpha=45^{\circ}$
$\theta=30^{\circ}$
$R=50 \mathrm{~m}$

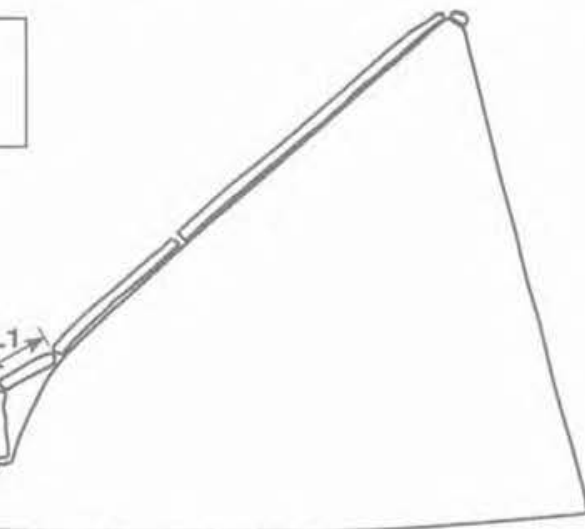

Schéma du modèle à la rupture. - Schematic of model at failure.

Fig. 10. - Essai sur la configuration 3. Fig. 10. - Test on configuration 3.

\section{ANALYSE DE L'ÉQUILIBRE LIMITE PAR LE LOGICIEL CURVBUK}

Le logiciel Curvbuk est un programme écrit en Turbo Pascal qui permet d'analyser l'équilibre limite d'un talus courbé à l'aide de la méthode de flambement à trois pivots. Les méthodes de flambement à trois pivots ont été utilisées par CHUGH (1977) et BEER (1982) pour l'analyse de la stabilité de toits de mines souterraines et par CAVERS (1981) pour la stabilité de talus de mines à ciel ouvert.
Le modèle géométrique analysé dans le cadre de cette étude est illustré à la figure 11 , toutefois le logiciel permet aussi d'analyser des configurations sensiblement différentes de celle illustrée. L'analyse à l'équilibre limite fait intervenir des forces inter-blocs à chacun des trois pivots, consécutives au poids propre des blocs, à la pression interstitielle d'eau agissant à l'arrière des blocs 1 et 2 et à des forces d'ancrage agissant sur ces mêmes blocs 1 et 2 (CANMET, 1986). Les différents paramètres nécessaires au logiciel sont les suivants (fig, 11) :

$\alpha$ : pendage des couches (degrés);

$\mathrm{R}$ : rayon de courbure $(\mathrm{m})$;

$\theta$ : angle au centre (degrés) ;

poids volumique de leau et de la roche $\left(\mathrm{kN} / \mathrm{m}^{3}\right)$;

$\mathrm{h}$ : hauteur de la nappe phréatique $(\mathrm{m})$;

$\mathrm{T}$ : épaisseur du banc $(\mathrm{m})$;

L1 : longueur du bloc $1(\mathrm{~m})$;

$\phi$ : angle de frottement inter-couche.

Le logiciel a été utilisé pour développer des abaques liant la hauteur du talus $\mathrm{H}$ à la longueur L1 du bloc 1 passif qui cause la rupture par flambage, ou plus précisément par expulsion à l'endroit du pivot 2. II est important de noter que la longueur L1 ne peut pas être visualisée sur le terrain car elle n'apparaît en réalité qu'au moment de la rupture. Toutefois, les résultats du logiciel sont donnés en fonction de ce paramètre qui a un effet important sur la hauteur limite du talus $\mathrm{H}$, lorsque tous les autres paramètres sont fixés.

Un exemple d'abaque pour le cas $\alpha=40^{\circ}, \theta=$ $30^{\circ}, \phi=25^{\circ}$ et diverses épaisseurs de banc T est présenté à la figure 12. De tels abaques constituent un résultat intéressant et inédit car on y observe que les courbes ont un minimum qui représente la hauteur maximale $\mathrm{H}$ que peut avoir le talus pour être stable.

La figure 13 représente un second type d'abaque développé à laide des résultats des simulations du

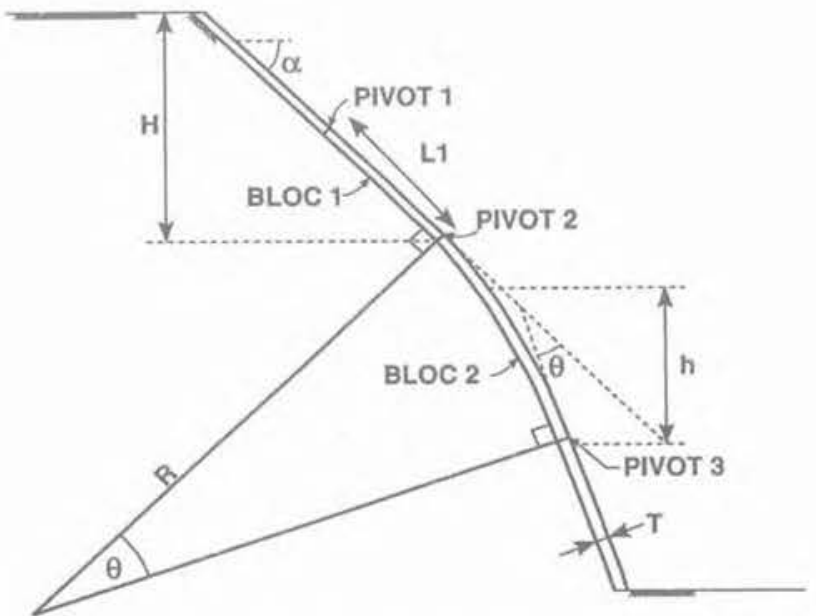

Fig. 11. - Modèle géométrique pour analyse par le logiciel Curvbuk.

Fig. 12. - Geometrical model for analysis with program Curvbuk. 


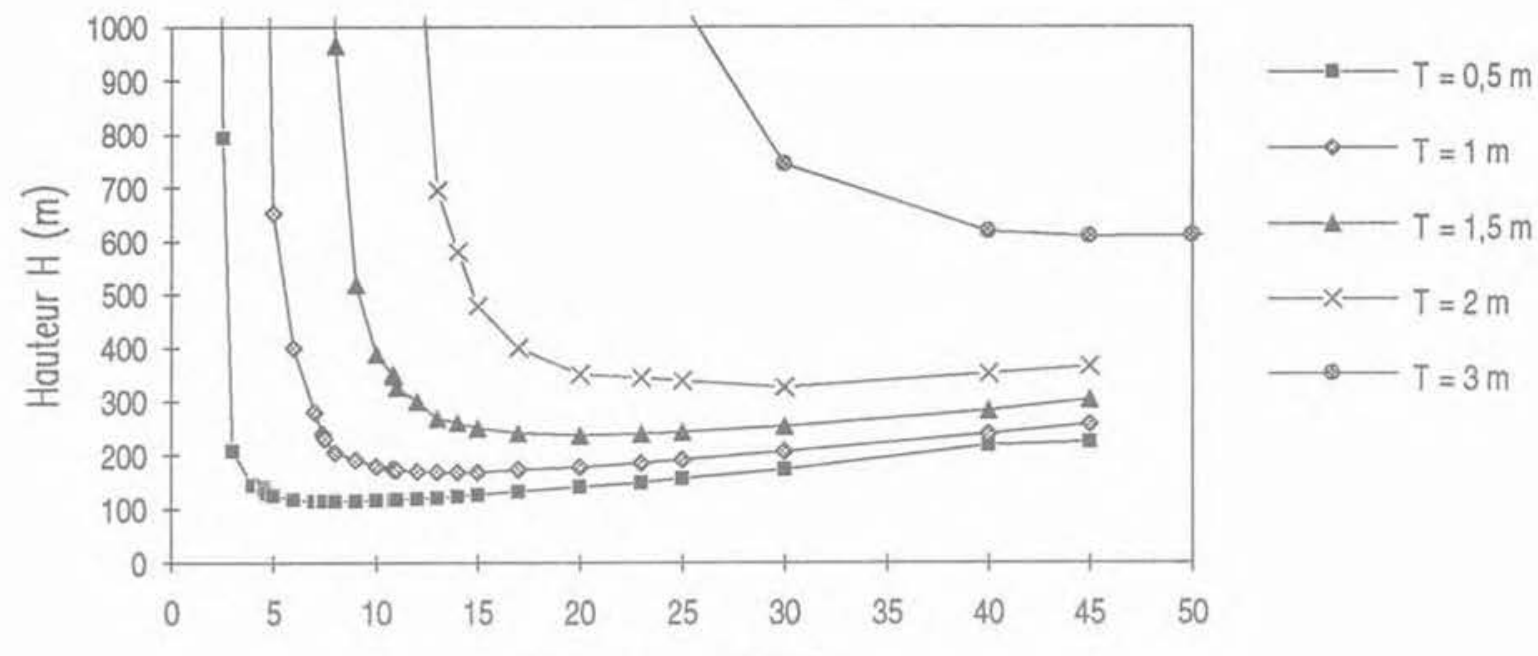

Longueur L1 (m)

Fig. 12. - Abaque de la hauteur $H$ en fonction de la longueur $L 1$ pour $\alpha=40^{\circ}, \theta=30^{\circ}, \phi=25^{\circ}$. Fig. 12. - Nomogram of height $H$ as a function of length $L 1$ for $\alpha=40^{\circ}, \theta=30^{\circ}, \phi=25^{\circ}$.

Comparaison de la Hauteur pour Théta $=30^{\circ}$.

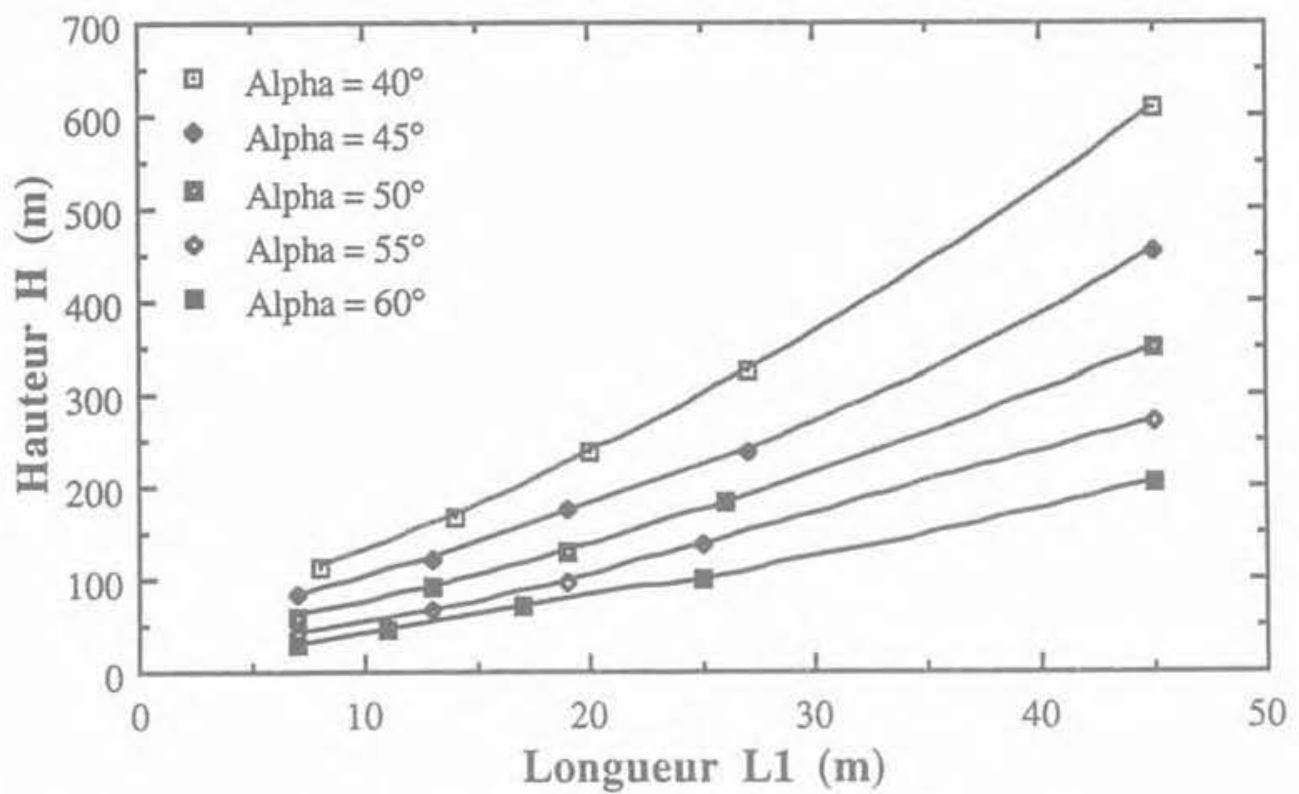

Fig. 13. - Minima des hauteurs pour $\theta=30^{\circ}$ et $\phi=25^{\circ}$ (de gauche à droite, les points correspondent à $T=0,5,1,1,5,2,3 \mathrm{~m})$.

Fig. 13. - Heights minimum for $\theta=30^{\circ}$ et $\phi=25^{\circ}$ (from left to right, points correspond to $T=0,5,1,1,5,2,3 \mathrm{ml}$.

logiciel. Cet abaque représente une synthèse d'abaques similaires à ceux de la figure 12 mais obtenus pour différentes valeurs de $\alpha$. Il représente les différents minima des hauteurs maximales que peut avoir le talus pour être stable, et ce pour différentes valeurs de $\alpha$ et avec $\theta$ et $\phi$ fixés. Il faut noter que chacun des cing points des courbes de l'abaque correspond à une épaisseur de banc $\mathrm{T}$ bien définie qui augmente de la gauche vers la droite.

A partir de l'abaque de la figure 13 , il est possible de déterminer une hauteur minimale du talus $\mathrm{H}$. Si cette hauteur est dépassée, les conditions de rupture par flambage des couches sont alors rencontrées.

\subsection{Confrontation des résultats du logiciel}

A titre d'exemple de l'utilisation des abaques et afin de corroborer les résultats du logiciel Curvbuk avec les essais par table à frottement, les configurations 3 à 5 et 9 à 11 de la figure 8 peuvent être confrontées aux prévisions du logiciel. Ces configurations ont un angle de courbure $\theta=30^{\circ}$ ce qui permet d'utiliser l'abaque de la figure 13 pour déterminer la hauteur minimale du talus au-delà de laquelle les conditions de rupture sont rencontrées. Bien qu'il n'ait pas été mesuré expérimentalement, l'angle $\phi$ s'appliquant aux modèles de la table à frottement a été supposé 
égal à $25^{\circ}$, ce qui correspond à l'abaque de la figure 13.

Dans le cas de la figuration 3 dont l'épaisseur des couches est de $1,8 \mathrm{~m}$, l'interpolation sur la courbe correspondant à langle $\alpha=45^{\circ}$ donne une hauteur minimale de talus de $220 \mathrm{~m}$. Par contre, le modèle qui représente une hauteur de talus réelle de $158 \mathrm{~m}$ a atteint la rupture ce qui n'est pas conforme aux prévisions du logiciel.

Les résultats des autres confrontations réalisées de la même façon sont résumés sur le tableau de la figure 14.

Il y apparaît qu'il y a une certaine concordance entre les résultats de Curvbuk et de la table à frottement. en particulier pour les configurations 9 à 11 . Toutefois, la confrontation montre bien qu'un nombre beaucoup plus grand d'essais par la table à frottement serait requis pour cerner de façon plus précise le seuil d'apparition de la rupture en fonction de l'épaisseur $T$ des couches et comparer ce seuil à celui prédit par le logiciel.

\subsection{Application du logiciel au cas de la mine de Grand-Baume}

Dans le cas de la mine de Grand-Baume, le pendage du talus est de l'ordre de $40^{\circ}$ (fig. 1). En supposant l'angle $\theta$ égal à $30^{\circ}$, on peut évaluer sur la figure 13 que la hauteur critique varie de $115 \mathrm{~m}$ pour une épaisseur des couches $T$ de $0,5 \mathrm{~m}$, à $170 \mathrm{~m}$ pour une épaisseur de $1 \mathrm{~m}$ et à $240 \mathrm{~m}$ pour une épaisseur de $1,5 \mathrm{~m}$.

Ces valeurs de hauteur critique correspondent tout à fait à la hauteur du talus qui sera atteinte lorsque l'exploitation aura atteint la profondeur maximale de $250 \mathrm{~m}$. De même, les épaisseurs de couche de $0,5 \mathrm{~m}$ à $1,5 \mathrm{~m}$ correspondent à l'ordre de grandeur observable à la mine. Ainsi, l'analyse par le logiciel fait apparaitre l'importance de cerner sur le terrain la valeur véritable de l'épaisseur des couches qui pourrait être mobilisée lors de l'amorce d'un glissement, puisque le passage de l'épaisseur de $0,5 \mathrm{~m}$ à $1,5 \mathrm{~m}$ suffit à rehausser la hauteur critique du talus de 115 $\mathrm{m}$ à $240 \mathrm{~m}$.

En réalité, lanalyse de la stabilité du talus par le logiciel Curvbuk a fait apparaître l'influence prépondérante de plusieurs autres paramètres en plus de l'épaisseur des couches sur la hauteur critique du talus. Le para- mètre $\theta$, aussi difficile à évaluer sur le terrain que l'épaisseur des couches, contribue à modifier de façon très significative la valeur de la hauteur critique. De même, la hauteur $h$ de la nappe phréatique dans le talus modifie de façon importante la valeur de la hauteur critique. Les forces d'ancrage des couches que le logiciel permet de simuler ont certainement une influence favorable sur la stabilité, toutefois elles n'ont pas été introduites dans les simulations réalisées au cours de cette étude.

\section{CONCLUSION}

L'utilisation de deux approches, d'abord par un modèle physique puis par un logiciel d'équilibre limite. pour l'étude de la stabilité du talus ouest de la fosse de Grand-Baume permet de mieux expliquer le mécanisme de flambage des couches qui pourrait se produire au moment où l'exploitation atteindra une profondeur suffisante pour dégager la charnière d'un pli couché qui délimite le talus.

Les modélisations réalisées permettent de mettre en lumière les caractéristiques géométriques du talus, soit l'épaisseur des couches et leur angle de courbure, qui doivent être estimées avec la meilleure précision possible afin de réduire l'incertitude associée à la détermination de la hauteur critique du talus. La meilleure connaissance de ces paramètres permettra de dimensionner de façon plus rationnelle le soutènement à utiliser pour stabiliser le talus.

Il semble important de pouvoir prendre en compte dans la modélisation, le renforcement, car ce renforcement existe et permet d'expliquer qu'actuellement aucune rupture n'ait été observée à Grand-Baume. L'étude réalisée a aussi permis de confronter les résultats obtenus en modèle réduit par la table à frottement avec ceux du logiciel. La confrontation n'a pu être que partielle à cause du faible nombre d'essais réalisés à l'aide de la table, toutefois la relative similitude des résultats et conclusions des deux approches obtenus jusqu'à maintenant justifie de continuer à utiliser les deux méthodes pour la poursuite de l'étude.

Finalement, l'étude réalisée a aussi permis de développer à l'aide du logiciel Curvbuk une approche à la préparation d'abaques d'évaluation de la stabilité des talus en présence de flambage des couches à trois pivots.

\begin{tabular}{|c|c|c|c|}
\hline Configuration & $\begin{array}{c}\text { Hauteur minimale requise } \\
\text { pour rupture }(\mathrm{m})\end{array}$ & Stabilité selon Curvbuk & $\begin{array}{c}\text { Stabilité selon table } \\
\text { à frottement (fig. 8) }\end{array}$ \\
\hline 3 & 220 & stable & rupture \\
4 & 390 & stable & stable \\
5 & 700 & stable & stable \\
9 & 130 & rupture & rupture \\
10 & 250 & stable & rupture \\
11 & 350 & stable & stable \\
\hline
\end{tabular}

Figure 14. - Confrontation des prévisions de stabilité par Curvbuk et par la table à frottement. Figure 14. - Comparison of stability predictions by Curvbuk and the friction table. 


\section{BIBLIOGRAPHIE}

BEER G., MEEK J.L. (1982), Design curves for roof and hangingwalls in bedded rock based on voussoir beams and plate solutions. Transactions institution of mining and metallurgy, Section A, Mining Industry, Vol. 91, p. 18-22.

BRAY J.W., GOODMAN R.E. (1981), The theory of base friction model. Int. J. Rock. Mech. Min. Sci. and Geomech. Abst., vol. 18, p. 453-468.

CANMET, Canada centre for mineral and energy technology (1986), Development of design criteria for steeply dipping footwall stability in mountain regions (UP.H-221). Final report by Hardy Associates (1978) Itd., DSS contract serial number: 1SQ85-00295, novembre 1986.

CAVERS D.S. (1981), Simple methods to analyze buckling of rock slopes. Rock Mechanics, Vol. 14/2, p. 87-104, 1981.

CAVERS D.S., SINGHAL R.K. (1988), Three hinge beam analysis for buckling of stratified rock strata. Proceedings of the first canadian conference on computer applications in the mineral industry, editors Fytas, Collins \& Singhal, 7-9 mars 1988, p. 269-275.
CHUGH A.K. (1977), Stability analysis of a jointed beam. International journal for numerical and analytical methods in geomechanics, vol. 1, p. 323-341.

EGGER P., GINDROZ C. (1979), Tunnels ancrés à faible profondeur. Etude comparative sur modèles physiques et mathématiques. Comptes rendus, 4 e Congrès de la Société internationale de mécanique des roches, vol. 2, p. 121-136.

HOBBS D.N. (1986), Scale model studies of strata movement around mine roadways. Apparatus, technique and some preliminary results. Int. J. Rock. Mech. Min. Sci., vol. 3, p. 101-127.

MANDEL J. (1962), Essais sur modèles réduits en mécanique des terrains. Etude des conditions de similitudes. Revue de l'industrie minérale, vol. 44, $n^{\circ} 9$, p. 611-620.

SOUKATCHOFF V., HANTZ D., MATHIEU E., PAQUETTE Y. (1991), Renforcement et contrôle des parements dans une mine à ciel ouvert de charbon. Comptes rendus, $7 \mathrm{e}$ Congrès de la Société internationale de mécanique des roches, Aachen, 19-21 septembre 1991.

SPANG R.M. (1976), Möglichkeiten und grenzen des base friction konzepts, Rock Mechanics, vol. 8/3, p. $185-198,1976$. 